\title{
A new view of quiet-Sun topology from Hinode/SOT
}

\author{
S. Régnier, C. E. Parnell, and A. L. Haynes \\ School of Mathematics and Statistics, University of St Andrews, St Andrews, Fife KY16 9SS, Scotland, UK \\ e-mail: stephane@mcs.st-andrews . ac .uk
}

Received 21 March 2008 / Accepted 8 May 2008

\begin{abstract}
Context. With the recent launch of the Hinode satellite our view of the nature and evolution of quiet-Sun regions has been improved. In light of the new high resolution observations, we revisit the study of the quiet Sun's topological nature.

Aims. Topology is a tool to explain the complexity of the magnetic field, the occurrence of reconnection processes, and the heating of the corona. This Letter aims to give new insights to these different topics.

Methods. Using a high-resolution Hinode/SOT observation of the line-of-sight magnetic field on the photosphere, we calculate the three dimensional magnetic field in the region above assuming a potential field. From the 3D field, we determine the existence of null points in the magnetic configuration.

Results. From this model of a continuous field, we find that the distribution of null points with height is significantly different from that reported in previous studies. In particular, the null points are mainly located above the bottom boundary layer in the photosphere $(54 \%)$ and in the chromosphere (44\%) with only a few null points in the corona (2\%). The density of null points (expressed as the ratio of the number of null points to the number of photospheric magnetic fragments) in the solar atmosphere is estimated to be between $3 \%$ and $8 \%$ depending on the method used to identify the number of magnetic fragments in the observed photosphere.

Conclusions. This study reveals that the heating of the corona by magnetic reconnection at coronal null points is unlikely. Our findings do not rule out the heating of the corona at other topological features. We also report the topological complexity of the chromosphere as strongly suggested by recent observations from Hinode/SOT.
\end{abstract}

Key words. Sun: magnetic fields - Sun: photosphere - Sun: chromosphere - Sun: corona

\section{Introduction}

The quiet-Sun magnetic field, the so-called magnetic carpet (Title \& Schrijver 1998), is thought to be divided into two main parts: closed field lines and open field lines (see e.g. Gabriel 1976). The closed field lines are low-lying intertwined field lines responsible for the complexity of the field. In particular, Close et al. (2003) revealed that $50 \%$ of the magnetic flux in the quietSun atmosphere is closed down within $2.5 \mathrm{Mm}$ above the solar surface, with almost $5-10 \%$ extending to $25 \mathrm{Mm}$ above the solar surface. It is assumed that this complexity, in addition to the fast recycling of the magnetic flux (Hagenaar et al. 2003; Close et al. 2005), is responsible for nano- and microflares that contribute to coronal heating (Parnell 2002). Thus, understanding the complex topology of the quiet-Sun magnetic field is key to understanding the coronal heating problem.

As known from decades of observations, the quiet-Sun magnetic field can be depicted by a large number of positive and negative sources. The source motions are characterized by emergence, coalescence, fragmentation and cancellation of magnetic polarities. These motions increase the complexity of the field. In order to analyse the complexity of the magnetic field, we study the existence and distribution of null points above an observed region of the quiet Sun. Even though null points are not the only relevant topological element in a magnetic configuration, they are a good proxy for the complexity of the field and for the existence of magnetic reconnection. Previous studies (Schrijver \& Title 2002; Longcope et al. 2003; Close et al. 2004) have tackled this topic with different approaches.
Schrijver \& Title (2002, hereafter ST02) modelled the quiet-Sun photosphere by point charges randomly distributed with a balanced magnetic flux. They also compared these with TRACE EUV images (Handy et al. 1999) and SOHO/MDI line-of-sight magnetograms (Scherrer et al. 1995). The coronal magnetic field was then defined analytically under the potential field assumption (i.e. no current present in the configuration) as detailed in Longcope (1996). These authors found that in the coronal volume there was approximately one null point per charge with $9 \%$ of the null points located in the corona (indicating that $91 \%$ of null points are photospheric null points). In the point charge approach used by ST02 the bottom boundary layer has a zero vertical component of the magnetic field everywhere except at each point charge. This assumption leads to many null points at the base. Their comparison with TRACE images showed that there is no link between the EUV brightenings and the number of null points, except for one location in their example. Longcope et al. (2003, hereafter LBP03) extended this study by producing a theory to determine the density of null points in random potential magnetic fields anchored in the photosphere. They tested their theory using a point charge approach and permitted an imbalance of flux thus producing open field lines. The authors found similar results to ST02 for the density of null points in the corona (above the photosphere). In addition LBP03 showed that the density of null points depends on how the sources are distributed in the photosphere and on the degree of imbalance of flux in the region. It is briefly mentioned in LBP03 that most of the photospheric null points are an artefact of the method used (no vertical field outside sources) and are proportional to 
the number of sources used in the realisation. In addition to simulated quiet-Sun data, Close et al. (2004, hereafter CPP04) used a SOHO/MDI photospheric magnetogram to study the complexity of the field. They converted the observed magnetogram into an array of point charges using a clumping approach to define the location and flux of the sources. In a large field-of-view of $240^{\prime \prime} \times 240^{\prime \prime}$, they found only 286 sources and a null point density of 0.038 in the corona (above the boundary).

In this Letter, we derive the null point density from a potential field extrapolation of a photospheric magnetogram obtained by Hinode/SOT. However, instead of assuming a point charge model, we consider a continuous magnetic field through the base. We compare our results with the statistical modeling of the quiet Sun by ST02, LBP03 and CPP04.

\section{Observation and model}

\subsection{Hinode/SOT data}

We use a line-of-sight magnetogram derived from the observed Stokes parameters $I$ and $V$ by the NFI (Narrowband Filter Imager) of the Hinode/SOT (Solar Optical Telescope, Tsuneta 2007). The observation was recorded on June 24, 2007 at 22:09 UT, and views a region slightly off the disc center. The NaI D1 resonance line at $5896 \AA$ is used. Although the core of the line $(< \pm 70 \mathrm{~m} \AA$ ) comes from chromospheric radiation (see e.g., Bruls et al. 1992), the NFI magnetogram is considered as a photospheric magnetogram since the measurements are made in the wings of the line at a spectral resolution of $90 \mathrm{~m} \AA$. The fieldof-view is $141^{\prime \prime} \times 161^{\prime \prime}(102 \mathrm{Mm} \times 116 \mathrm{Mm})$ with a pixel width of $0.16^{\prime \prime}(880 \times 1012$ pixels $)$. The pixel width of SOT/NFI is 3.8 times better than that for high resolution observations taken by SOHO/MDI. The analysis, reduction and calibration of this dataset are detailed in Parnell et al. (2008).

In Fig. 1, we plot the distribution of the line-of-sight magnetic field in a quiet-Sun region as observed by SOT/NFI. The maximum absolute field strength is $717 \mathrm{G}$. The net flux is $3.58 \times 10^{20} \mathrm{Mx}$ and the total unsigned flux is $1.5 \times 10^{21} \mathrm{Mx}$. The imbalance of magnetic flux is then $\Delta \phi=+24 \%$. The sign indicates that there is an excess of positive magnetic flux.

\subsection{Potential field model}

Assuming that the coronal magnetic field is essentially in equilibrium, neglecting the gas pressure and gravity forces, as well as the currents flowing along field lines, we obtain the potential field assumption satisfying $\boldsymbol{\nabla} \wedge \boldsymbol{B}=\mathbf{0}$. The potential field corresponds to the minimum energy state. Potential field extrapolation methods require only the vertical (resp. radial) magnetic field component in Cartesian geometry (resp. spherical geometry) at the bottom boundary layer (e.g. Schmidt 1964; Semel \& Rayrole 1968). This is a well-posed boundary value problem giving a unique solution for prescribed boundary conditions on all boundaries. In the past, several types of field distributions have been used as the base boundary condition: (i) the point charge method which permits an analytical expression for the coronal field to be derived assuming that each magnetic fragment is reduced to a single point (e.g., Longcope 1996); (ii) the discrete source method for which the polarities have a finite width but there is no field outside; (iii) the continuous field method where the observed magnetic field is discretised and then extrapolated numerically within a finite box. We here apply the last method using the Hinode/SOT data detailed in the previous Section as a bottom boundary condition (defined on a smaller

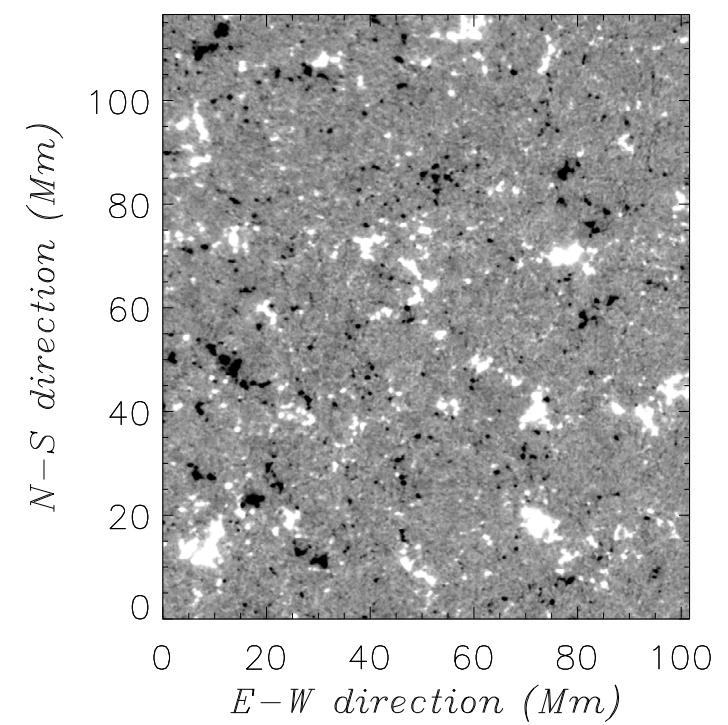

Fig. 1. Distribution of the line-of-sight magnetic field on the photosphere observed by Hinode/SOT NFI on June 24, 2007 at 22:09 UT. The image is saturated at $\pm 50 \mathrm{G}$. Positive (negative) polarities are white (black). North is up.

grid of $292 \times 336$ pixels due to computational limitations). As the magnetic flux is not balanced, we impose open boundary conditions on the sides and top of the box allowing field lines to enter or leave the box. Due to the assumed high complexity of the quiet-Sun field near the photosphere, we use a stretched grid in the vertical direction with a finer grid near the photosphere.

\subsection{Null point finder}

A classical method to find null points in a field configuration is based on the computation of the Poincaré index (Greene 1992). Unfortunately the method fails for weakly and strongly nonlinear fields (Haynes \& Parnell 2007). Recently, a more reliable and stable method for linear and moderately nonlinear fields has been developed: the trilinear method (Haynes \& Parnell 2007). By comparison to Greene's method, the trilinear method is more appropriate for numerical fields whilst Greene's method is only really suitable for analytical fields where any grid can be easily made finer if the method fails. The trilinear method has been successfully applied to different numerical experiments (Haynes \& Parnell 2007). In order to apply this method, the considered vector field has to be linear or moderately nonlinear within a cell. Here we have had to use a stretched grid in the $z$ direction with smaller grid spacing near the photosphere to ensure that the complexity of the field is well resolved in the low atmosphere.

\section{Discussion and conclusions}

We extrapolate the coronal field under the potential field assumption from the photospheric line-of-sight component provided by Hinode/SOT data (Fig. 1). Then we look for null points in the three dimensional magnetic configuration using the trilinear method.

In Fig. 2, we plot the spatial distribution of the null points in the $x y$-plane (parallel to the photospheric plane). We find 80 null points distributed in height from the bottom boundary to about $5 \mathrm{Mm}$ above which no null points are found. None of the null points lie exactly on the bottom boundary. The spatial distribution in the $x y$-plane is seemingly random, in particular there exist 


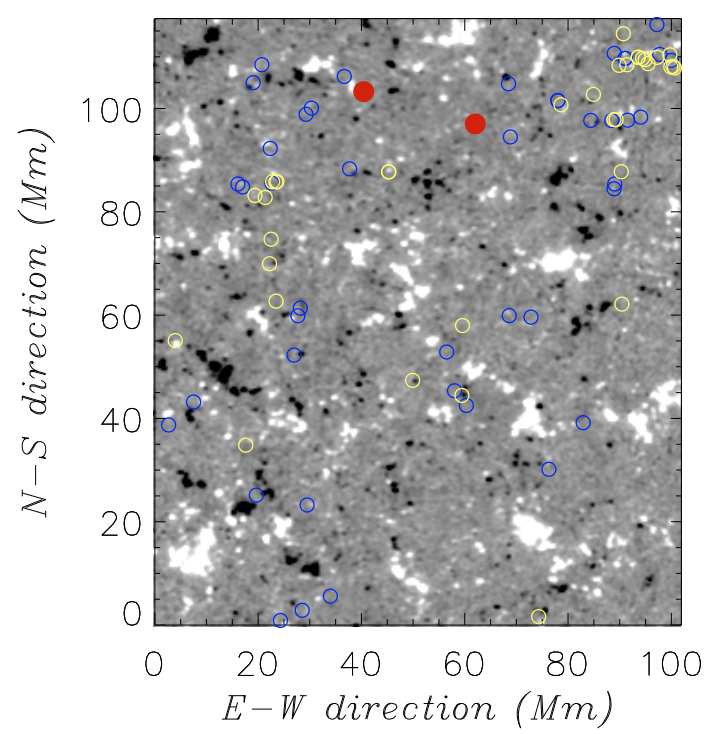

Fig. 2. Location of the null points in the photosphere (blue circles), in the chromosphere (yellow circles) and in the corona (filled red circles). Background image: line-of-sight SOT magnetogram used for the potential field extrapolation (defined on a smaller grid of $292 \times 336$ pixels due to computational limitations) saturated at $\pm 50 \mathrm{G}$.

areas as large as a network cell $(20 \mathrm{Mm} \times 20 \mathrm{Mm})$ without null points. Few null points lie above concentrations of flux.

We now assume a typical solar atmosphere: the photosphere from 0 to $1 \mathrm{Mm}$, the chromosphere from 1 to $3.5 \mathrm{Mm}$, and then the corona. This atmosphere is depicted in Fig. 3 left by the horizontal solid lines. We assume that the height of formation of the NaI D1 line provided by SOT/NFI is at the base of photosphere. Under these assumptions, we find:

- 43 null points (54\%) in the photosphere;

- 35 null points (44\%) in the chromosphere;

- 2 null points $(2 \%)$ in the corona.

As shown in Fig. 3 right, there is a uniform distribution of null points below $2 \mathrm{Mm}$ (approximately 70-95\% of the characteristic source separation) whilst the distribution of all atmospheric null points is well fitted by an exponential distribution with a characteristic length of $1.04 \mathrm{Mm}$ which is about half of the source separation as in ST02.

We define the density of null points as the ratio of the number of the null points to magnetic fragments in order to compare our results with ST02, LBP03 and CPP04. To derive this quantity, we need to determine the number of magnetic fragments in the quiet-Sun region. There are several methods to identify the fragments as reviewed by DeForest et al. (2007). For comparison, we only use two methods: the clumping approach and the peak approach. Following Parnell et al. (2008), there are more than one thousand contiguous magnetic flux fragments assuming a threshold of $18 \mathrm{G}$ in the observed quiet-Sun region using the clumping approach, giving a density of null points less than $7.5 \%$ (1063 fragments). The peak approach with a threshold of $18 \mathrm{G}$ gives 2680 fragments leading to a density of null points of $3 \%$.

The results obtained here from the extrapolation of a continuous photospheric distribution may be compared to those found using the point charge method of simulated data used by ST02, LBP03 and CPP04 (not reported here). The estimated densities of null points are summarised in Table 1 . With the point charge method used by ST02, the density of null points on the
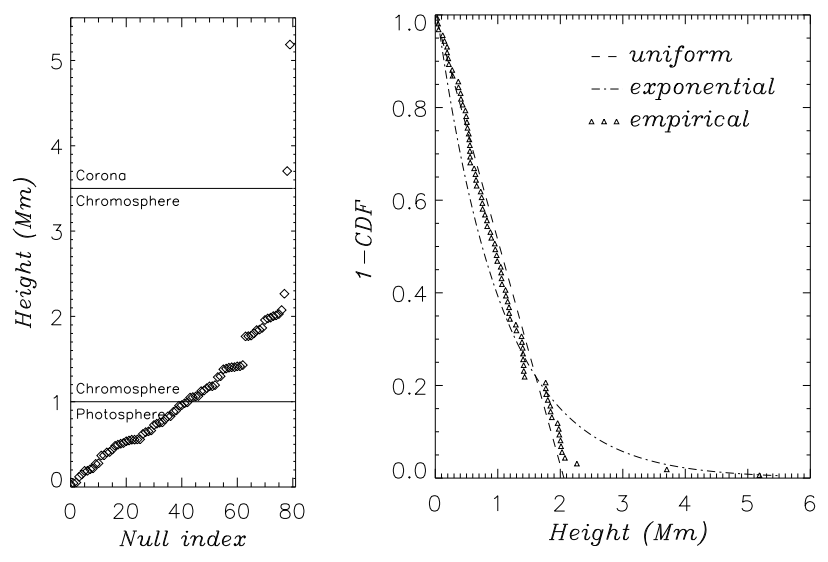

Fig. 3. Left: distribution of null points with height (diamonds). 43 null points are in the photosphere below $1 \mathrm{Mm}, 35$ null points in chromosphere between 1 and $3.5 \mathrm{Mm}$, and 2 null points are in the corona. Right: survival functions (1-CDF, Cumulative Distribution Function) for the distribution of null point heights (triangles), the exponential fit for the atmospheric null points (dot-dashed curve) and the uniform fit for the photospheric and chromospheric null points (dashed curve).

Table 1. Summary of the null point density depending on the location in the solar atmosphere for the different methods (see text for detail).

\begin{tabular}{lccccc}
\hline \hline density & ST02 & LBP03 & CPP04 & \multicolumn{2}{c}{ This work } \\
\hline photosphere & & & clump & clump & peak \\
$\quad$ boundary & 1.001 & N/A & 1.05 & 0 & 0 \\
above & N/A & N/A & N/A & 0.04 & 0.016 \\
chromosphere & N/A & N/A & N/A & 0.033 & 0.013 \\
corona & 0.093 & $0.07-0.09$ & 0.038 & 0.002 & 0.0007 \\
total atmos. & 0.093 & $0.07-0.09$ & 0.038 & 0.075 & 0.03 \\
\hline grand total & 1.094 & $0.07-0.09$ & 1.088 & 0.075 & 0.03 \\
\hline
\end{tabular}

photosphere (bottom boundary layer) is about one null point per charge, and $9 \%$ of null points in the corona (above the bottom boundary). In LBP03, it is not possible to derive the density of null points on the bottom layer due to the definition of the random distribution of point charge sources although there will be many, but the density in the corona is between 7-9\% Clearly the density of null points estimated using the point charge approach and the estimate made using magnetic fragments determined from the clumping identification method in a continuous field can be reconciled by simply removing the base boundary layer from consideration. If this is done, the density of null points in the solar atmosphere (photosphere + chromosphere + corona) is about 7-9\%. Nevertheless if the number of sources is derived from the peak method, then the density of null points is much less $(3 \%)$, which is comparable to the density obtained with observed magnetogram by CPP04. Note that compared to our study with the peak approach, CPP04 used a field-of-view five times larger but involving 10 times fewer sources. Clearly, the comparison of the clumping results from this work and CPP04s results suggests that the spatial density of null points may also vary with resolution. This will be investigated in detail in a subsequent paper. This suggests that the density of null points is not an appropriate quantity to describe the complexity of an observed quietSun region as it relies on a magnetic field model and a definition of magnetic fragments.

As pointed out by ST02, the topology of the quiet Sun is important in understanding the possible heating mechanisms of the corona. By comparing EUV images and photospheric 
magnetograms, ST02 showed that there is no correlation between the coronal EUV brightenings (from the FeXII line at $195 \AA$ ) and the location of null points, with the exception of one point. From our study, we see that only 2 null points are in the corona and are potentially observable in such a hot corona. Most of the null points should be observable in the chromosphere. The complexity of the chromosphere was recently highlighted by the CaII images from Hinode/SOT (see e.g., De Pontieu et al. 2007). Due to the low spatial density of null points in the corona, the heating of the corona by magnetic reconnection at null points located in the corona can then be excluded. Nevertheless reconnection can occur at different locations with (e.g., separators, separatrix surfaces) or without (e.g., hyperbolic flux tubes) null points.

In a forthcoming paper, we will develop the study of the topology of the quiet-Sun field by looking at the connectivity of field lines, the stability and nature of null points and their time evolution. This study is important to understand the dynamic behaviour of heating in a complex topology.

Acknowledgements. We thank the referee for his/her comments which helped to improve this Letter. We thank the UK STFC for financial support (STFC RG). C.E.P. is funded by STFC on an Advanced Fellowship. Hinode is a Japanese mission developed and launched by ISAS/JAXA, with NAOJ as domestic partner and NASA and STFC (UK) as international partners. It is operated by these agencies in co-operation with ESA and NSC (Norway). The computations have been done using the XTRAPOL code developed by T. Amari (Ecole Polytechnique, France). We also acknowledge the financial support by the European Commission through the SOLAIRE network (MTRN-CT-2006035484).

\section{References}

Bruls, J. H. M. J., Rutten, R. J., \& Shchukina, N. G. 1992, A\&A, 265, 237 Close, R. M., Parnell, C. E., Mackay, D. H., \& Priest, E. R. 2003, Sol. Phys., 212,251

Close, R. M., Parnell, C. E., \& Priest, E. R. 2004, Sol. Phys., 225, 21

Close, R. M., Parnell, C. E., Longcope, D. W., \& Priest, E. R. 2005, Sol. Phys., 231, 45

De Pontieu, B., McIntosh, S. W., Hansteen, V. H., et al. 2007, Publ. Astron. Soc. Japan, 59, S655

DeForest, C. E., Hagenaar, H. J., Lamb, D. A., Parnell, C. E., \& Welsch, B. T. 2007, ApJ, 666, 576

Gabriel, A. H. 1976, R. Soc. London Phil. Trans. Ser., 281, 339

Greene, J. M. 1992, J. Comp. Phys., 98, 194

Hagenaar, H. J., Schrijver, C. J., \& Title, A. M. 2003, ApJ, 584, 1107

Handy, B. N., Acton, L. W., Kankelborg, C. C., et al. 1999, Sol. Phys., 187, 229

Haynes, A. L., \& Parnell, C. E. 2007, Phys. Plasmas, 14, 2107

Longcope, D. W. 1996, Sol. Phys., 169, 91

Longcope, D. W., Brown, D. S., \& Priest, E. R. 2003, Phys. Plasmas, 10, 3321

Parnell, C. E. 2002, MNRAS, 335, 389

Parnell, C. E., DeForest, C. E., Hagenaar, H. J., Lamb, D. A., \& Welsch, B. T. 2008, ApJ

Scherrer, P. H., Bogart, R. S., Bush, R. I., et al. 1995, Sol. Phys., 162, 129

Schmidt, H. U. 1964, in The Physics of Solar Flares, ed. W. N. Hess, 107

Schrijver, C. J., \& Title, A. M. 2002, Sol. Phys., 207, 223

Semel, M., \& Rayrole, J. 1968, in Structure and Development of Solar Active Regions, ed. K. O. Kiepenheuer, IAU Symp., 35, 134

Title, A. M., \& Schrijver, C. J. 1998, in Cool Stars, Stellar Systems, and the Sun, ed. R. A. Donahue, \& J. A. Bookbinder, ASP Conf. Ser., 154, 345

Tsuneta, S. 2007, ArXiv e-prints, 711 